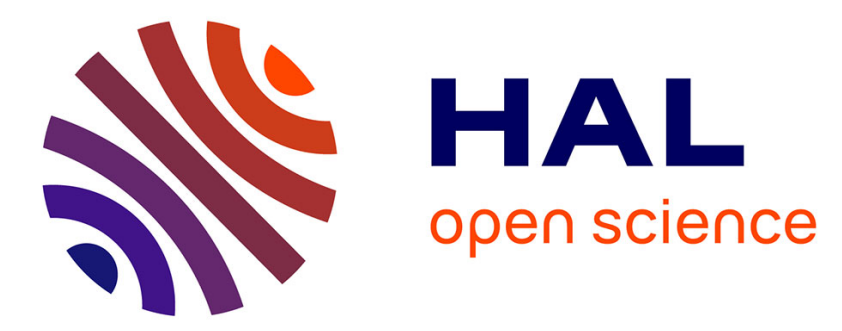

\title{
Théorie de la capture sélective des neutrons lents par certains noyaux
}

\author{
Francis Perrin, Walter M. Elsasser
}

\section{To cite this version:}

Francis Perrin, Walter M. Elsasser. Théorie de la capture sélective des neutrons lents par certains noyaux. J. Phys. Radium, 1935, 6 (5), pp.194-202. 10.1051/jphysrad:0193500605019400 . jpa00233320

\section{HAL Id: jpa-00233320 https://hal.science/jpa-00233320}

Submitted on 4 Feb 2008

HAL is a multi-disciplinary open access archive for the deposit and dissemination of scientific research documents, whether they are published or not. The documents may come from teaching and research institutions in France or abroad, or from public or private research centers.
L'archive ouverte pluridisciplinaire HAL, est destinée au dépôt et à la diffusion de documents scientifiques de niveau recherche, publiés ou non, émanant des établissements d'enseignement et de recherche français ou étrangers, des laboratoires publics ou privés. 


\title{
THÉORIE DE LA GAPTURE SÉLEGTIVE DES NEUTRONS LENTS PAR GERTAINS NOYAUX
}

Par Francis Perrin et Walter M. ElSasser.

(Institut Henri Poincaré, Paris.)

\begin{abstract}
Sommaire. - Ce travail met en évidence un mécanisme de résonance pouvant rendre compte des valeurs très grandes des sections efficaces de capture des neutrons lents observées pour quelques éléments par Fermi (plus de mille fois la section nucléaire), sans supposer d’action à distance entre les neutrons et les noyaux ( $\left.{ }^{1}\right)$

Pour une interaction limitée au domaine nucléaire, les états stationnaires possibles du neu'ron sont en nombre fini, et il peut arriver que l'un d'eux corresponde à une énergie de liaison nulle (niveau d'énergie " superficiel »). Les grandes sections efficaces pour la capture d'un neutron, avec émission d'un photon $\gamma$, se présenteront s'il existe dans le noyau un niveau neutronique libre de type $P$ énergétiquement profond, sur lequel se fera la capture, et un niveau superficiel de type $S$ déterminant la résonance.

Le calcul complet est fait dans l'hypothèse schématique d'un potentiel d'interaction constant $\varepsilon_{0}$ limité à une sphère de rayon $r_{0}$. Il donne des sections efficaces $\sigma_{c}$ négligeables sauf pour les neutrons d'énergie $\omega$
\end{abstract} très petite, pour lesquels on a aproximativement

$$
\sigma_{c} \sim \frac{\varepsilon_{n}}{\omega+\varepsilon_{0} \cos ^{2} \rho_{0}} \sqrt{\frac{\varepsilon_{0}}{\omega}} \cdot 10-30 \mathrm{~cm}^{2}
$$

avec $\rho_{0}=2 \pi h^{-1} \sqrt{2 \mu \varepsilon_{0}} r_{0}$ et $\varepsilon_{0} \sim r_{0}^{-2.3 .10-24}$ MVe (l'énergie de liaison étant de l'ordre de $\varepsilon_{0} / 2$ ). La résonance se produit pour $\cos \rho_{0}=0$; si elle est seulement approchée, la section efficace de capture sera grande pour les neutrons très lents, et proportionnelle à l'inverse de la vilesse.

La résonance qui détermine les grandes sections efficaces lient au ccmportement de l'onde neutronique incidente dans la région du noyau; elle peut do ne jouer aussi pour une capture du neutron associée à l'émission d'une particule matérielle mais alors l'existence d'un niveau $/$ ' profond n'est plus nécessaire'.

Enfin l'étude d'un polentiol quelconque à décıoissance rapide, montre l'existence générale des phénomènes de résonance analysés ici dans le cas schématique du " trou " de potentiel.

La formation d'éléments radioactifs négatogènes, par l'action des neutrons sur la matière, se produit selon deux types principaux de réaclions nucléaires, suivant que la capture du neutron incident est ou non accompagnée de la réémission immédiate d'une particule matérielle (réaction de substitution ou d'addition;.

Le deuxième mécanisme (capture simple) que nous étudierons principalement ici, est mis en évidence par l'isotopie de l'élément radioactif formé et de l'élément irradié; il doit être accompagné de l'émission d'un rayonnement $\gamma$ correspondant à l'énergie de fixation des neutrons augmentée de l'énergie cinétique qui disparait lors de la capture. Les sections efficaces des noyaux pour une telle capture simple des neutrons émis directement par les sources usuelles, sont de l'ordre de $10^{-25} \mathrm{~cm}^{2}$, c’est-à-dire peu inférieures aux sections des domaines nucléaires déterminés par la dispersion anor-

( ${ }^{1}$ Cne note contenant les résultats principaux de ce travail a déjà été publiée :

F. Perrix et W. M. Elsasser C. R. Ac. Sc., 4 février 1935̆, 200, p. 450 . male ou l'émission des particules $\alpha\left({ }^{2}\right)$. La probabilité de capture d'un neutron qui traverse un noyau doit donc être assez grande, ce qui est difficile à comprendre, car, d’après la théorie de l'émission du rayonnement $\%$ par les particules nucléaires massives, la durée de la traversée d'un noyau, par un neutron projeté par une transmutation, semble devoir être très petite par rapport à la durée moyenne relative à l'émission d'un photon, même s'il représente une énergie d'une dizaine de mégavolts électron.

D'autre part, lorsque intervient le mécanisme de capture simple des neutrons, avec formation d'un élément radioactif isotope du corps irradié, on constate que l'interposition d'une substance hydrogénée (eau, paraffine) dans le rayonnement incident augmente beaucoup l'activité produite (effet Fermi). Cet effet remarquable ne semble pouvoir résulter que d'un grand ralentissement d'une partie des neutrons incidents par des chocs sur des protons de la substance

(2) Fermi, Amaldi, d’Agostivo, Rasetti, Segré. Proc. Roy. Soc, A, 1934, 146, p. 483 . 
No 5. THÉORIE DE LA CAPTURE SÉlEGTIVE DES

hydrogénée; un neutron perdant en moyenne la moitié de son énergie cinétique lors d'un choc sur un proton, il suffit d'une vingtaine de chocs, si son énergie est initialement de l'ordre d'un mégavoltélectron, pour qu'elle soit devenue comparable à l'énergie d'agitation thermique des protons rencontrés. Les neutrons lents, ainsi obtenus et décelés, sont absorbés de façon très différente, et sans aucune variation régulière en fonction du nombre atomique, par les divers éléments.

Certains, comme le plomb ou le carbone, sont pour eux presque tout à fait (transparents », tandis que d'autres, les absorbent fortement sous une épaisseur très faible, en donnant ou non naissance à des corps radioactifs. Pour certains éléments (tels le bore et le cadmium) la section efficace des noyaux pour la capture des neutrons lents atteint des valeurs de l'ordre de $10^{-21} \mathrm{~cm}^{2}$ et même de $10^{-20} \mathrm{~cm}^{2}$, soit de mille à dix mille fois la section des domaines nucléaires; ces éléments ne présentent d'ailleurs aucune absorption exceptionnelle pour les neutrons rapides. L'absorption considérable des neutrons lents apparait ainsi comme sélective, et déterminée par un caractère particulier de structure des noyaux absorbants.

Les succès déjà obtenus par l'application de la mécanique ondulatoire aux particules nucléaires massives, malgré notre ignorance de leurs lois d'interaction, incitent à tenter d'expliquer ces phénomènes nouveaux en les rattachant à des propriétés générales de systèmes quantifiés simples schématisant les noyaux.

Il est utile pour cela de considérer, en même temps que le phénomène de capture d'un neutron avec émission d'un photon, le phénomène inverse d'arrachement photoélectrique d'un neutron à un noyau par un rayonnement $\gamma$, phénomène découvert par Chadwick dans le cas du deutohydrogène et observé depuis aussi pour le glucinium.

Le principe général de microréversibilité des transformations quantiques détermine le rapport des sections efficaces $\sigma_{c}$ et $\sigma_{e}$ relatives aux deux phénomènes inverses de capture d'un neutron de vitesse $u$ par un noyau $A_{0}$ pour former un noyau $A_{1}$ avec émission $\mathrm{d}$ un photon $h v$, et d'arrachement photoélectrique d'un neutron au noyau $A_{1}$ par un rayonnement $\gamma$ de fréquence v. Pour étudier ces mécanismes on peut se borner à considérer le mouvement relatif du neutron par rapport au noyau $A_{0}$ de masse $\boldsymbol{M}_{0}$, à condition de remplacer la masse vraie $m$ du neutron par la masse réduite

$$
\mu=\frac{m M_{0}}{m+M_{0}} \text {. }
$$

L'équation de conservation de l'énergie s'écrit ainsi

$$
\frac{1}{2} \mu u^{2}+\varepsilon=h \nu \quad \text { ou } \quad \omega+\varepsilon=h \nu
$$

$\varepsilon$ étant l'énergie de liaison du neutron sur le noyau $A_{0}$, et $\omega$ l'énergie cinétique dissipée lors de la capture. L'égale probabilité des mécanismes exactementinverses s'exprime en dénombrant les états quantiques corres-
NEUTRONS LENTS PAR CERTAINS NOYAUX

195

pondant à un petit intervalle d'énergie, et l'on trouve (') en tenant compte du spin $1 / 2$ du neutron et des deux états possibles de polarisation d'un photon

$2\left(2 j_{0}+1\right) 4 \pi(\mu . u)^{2} \mathrm{~d}(\mu u) u_{i_{c}}$

$$
=2\left(2 j_{1}+1\right) 4 \pi\left(\frac{h \nu}{c}\right)^{2} \mathrm{~d}\left(\begin{array}{c}
h \nu \\
c
\end{array}\right) c \sigma_{e}
$$

$j_{0}$ et $j_{1}$ étant les spins des noyaux $A_{v}$ et $A_{1}$. Comme d'après la relation (2) on a

$$
\mu u \mathrm{~d} u=h \mathrm{~d} v
$$

il reste

$$
\sigma_{c}=\frac{2 j_{1}+1}{2 j_{0}+1} \frac{(h \nu)^{2}}{\mu c^{2} \cdot \psi \cdot u^{2}} \sigma_{e}=\frac{1}{2} \frac{2 j_{1}+1}{2 j_{0}+1} \frac{(\varepsilon+\omega)^{2}}{\mu c^{2} \omega} \sigma_{e}
$$

Nous utiliserons cette formule pour calculer la section efficace relative à la capture d'un neutron, en déduisant $\sigma_{e}$ de la théorie quantique générale de l'effet photoélectrique. Pour un système petit par rapport à la longueur d'onde $\lambda$ de la lumière excitatrice, cette théorie donne

$$
\sigma_{e}=\frac{8 \pi^{3} v}{c}\left|\varkappa_{Z O E}\right|^{2}
$$

$\mathfrak{K}_{Z O E}$ étant l'élément de matrice de la composante $\mathfrak{K}_{Z}$ du moment électrique $\overrightarrow{\jmath \mathfrak{r}}$ du système de particules par rapport à son centre d'inertie, suivant la direction du champ électrique de l'onde incidente, pour la transition entre l'état initial des particules liées et l'état final de dissociation, normalisé pour une représentation en fonction de l'énergie du spectre continu dont il fait partie. Pour un neutron à une distance $r$ du noyau $A_{0}$ de charge $Z e$, le moment $\overrightarrow{\partial r}$ a comme valeur

$$
\overrightarrow{\jmath \mathfrak{L}}=\mu \frac{Z e}{M_{0}} \vec{r}
$$

On aura donc

$$
c_{e}=\frac{8 \pi^{3 y}}{c} \mu^{2}\left(\frac{Z e}{\bar{I}_{0}}\right)^{2}\left|z_{O E}\right|^{2} .
$$

Il faut noter cependant que ces formules ne sont valables que si elles donnent pour l'effet photoélectrique un rayon efficace petit par rapport à la longueur d'onde $\curlywedge$, divisée par $2 \pi$, c'est-à-dire si

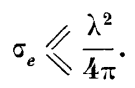

Théorie du deuton de Bethe et Peierls. - Ces auteurs $\left(^{2}\right)$ ont réussi à calculer complètement à partir des formules précédentes, les sections efficaces $\sigma_{e}$ et $\sigma$ dans le cas de la décomposition ou de la formation du deuton $\left({ }_{1}^{0} \mathrm{H}={ }_{1}^{1} \mathrm{H}+{ }_{0}^{1} u+h v\right)$, en admettant que l'in-

(1) Cf. Betee et Peierls. Proc. Roy. Soc., A, 1935, 148, p. 146. (') Loc. cut 
teraction entre un proton et un neutron est très intense, mais limitée à une région très pelite (potentiel de l ordre de $10^{8}$ eV pour une dislance inférieure à $10^{-13} \mathrm{~cm}$ ). Cette hypothèse, justifiée par l'explication qu'elle permet de la grande différence des énergies de liaison du deuton et de l'hélion (1), impose l'existence đ’un seul état stationnaire possible pour le système proton neutron, état correspondant à un moment de circulation nul ( $l=0$, état $S)$. L'élément de matrice $z_{O E}$, et par suite $\sigma_{e}$, est alors déterminé en fonction de l'énergie de liaison, qui correspond par ailleurs au seuil de la dissociation photoélectrique. La valeur obtenue ainsi pour la section efficace $\sigma_{e}$ est à peu près en accord avec la détermination expérimentale de Chadwick.

La section efficace $\sigma_{e}$, pour le phénomène inverse de capture d'un neutron par un proton, déduite de ce calcul à l'aide de la formule (3) atteint un maximum de seulement $2.10^{-29} \mathrm{~cm}^{2}$ pour des neutrons incidents ayant une énergie d'environ $4.10^{6} \mathrm{eV}$ et tend vers zéro quand l'énergie des neutrons tend elle-même vers zéro. Ce résultat explique bien l'absence d'absorption vraie des neutrons rapides ou lents par l'hydrogène. mais ne permet pas de comprendre l'existence dans d'autres cas, de sections efficaces de capture très grandes pour des neulrons lents.

Liaisond'un neutron sur un noyauquelconque. - Nous ignorons les lois d'action entre les neutrons et les noyaux atomiques ; ceperidant la plupart des faits observés suggèrent que leur interaction devient rapidement négligeable hors du domaine nucléaire déterminé par l'apparition d'écarts à la loi de Coulomb pour les particules $\alpha$ (rayon de l'ord re de quelque $10^{-13} \mathrm{~cm}$ ) Seules les grandes sections de capture observées par Fermi pourraient faire supposer une interaction s'étendant à une dislance beaucoup plus grande, mais nous nous proposons de montrer qu'on peut en rendre compte sans faire une telle hypothèse. l'our plus de simplicité.négligeantla complexité des noyaux,nousarlmettrons icique l'interaclion, limitée au domaine nucléaire, résulte d'un potenliel ordinaire entre deux particules; il est vraisemblable que l’essentiel des résultals que nous obtiendrons ainsi reste au moins qualilativement vrai pour d'autres types d'interaction (énergie d'échange).

Nous devons d abord rappeler qu'un potentiel d'attraction tendant rapidement vers zéro quand la distance augmente conduit à un schéma de niveaux énergéliques tout à fait différent de cclui qui résulte d'un potentiel coulombien altractif. L'étude complète a pu être faite dans le cas simple d'un potentiel sphérique constant limité brusquement (voir l'appendice) et, du moins pour les niveaux $S$, dans le cas d'un potentiel décroissant exponentiellement $\left({ }^{2}\right)$. Les résultats sont analogues dans ces deux cas et sont sans doute va-

(1) E. Wigner. Phys. Rev., 1933, 43, $2 \% 2$.

(ะ) Masser et Mohr. Proc. Roy. Soc., A, 1934, 141, 434 et 1935 148, 206 . lables pour tout potentiel décroissant suffisamment vite.

Dans un tel potentiel, le nombre des états stationnaires discrets est fini; il peut même n'en exister aucun si la profondeur du " trou " de potentiel, ou son étendue, sont insuffisantes. C'est ainsi que, comme nous l'avons dit, il n'existe probablement qu'un tel niveau, de type $S$, pour les deutons. Pour une interaction moins étroitement limitée, il pourra exister quelques élats, certains correspondant à un moment de circulation non nul $(l=1$, états $P, \ldots)$.

Or, on voit facilement que la section efficace relative à la capture d'un neutron par un proton tend vers zéro avec l'énergie du neutron incident, parce que la capture se fait sur un niveau $S$, et que l'émission du photon, qui exige une variation d'une unité du moment cinétique d'orbite $(\Delta l= \pm 1)$, ne peut par suite se produire que si le neutron incident a, par rapport au proton, un moment unité $h / 2 \pi$, ce qui implique qu'il passe à une distance d'autant plus grande qu'il va plus lentement.

Au contraire, si la capture se produit sur un niveau $P$, le neutron pourra avoir un moment cinétique initial nul par rapport au noyau. Nous devrons donc faire le culcul de la section efficace dans un tel cas.

D'autre part, dans la région d'interaction, la grandeur des fonctions d'onde normalisées du spectre continu varie très rapidement en fonction de l'énergie du neutron. Ces variations, d'allure périodique, représentent une surte de prolongement du spectre discontinu fini à travers le spectre continu; les maxima peuvent être considérés comme des états stationnaires virtuels du système; ils existent pour les états $s$, mais sont plus aigus lorsque le moment cinétique n'est pas nul (états $p, d, \ldots)$ à cause de la ( barrière » de potentiel qui correspond à la force centrifuge $\left(^{(1)}\right.$.

Les alternances d'intensité des fonctions d'onde du spectre continu dans l'intérieur du noyau ont pour conséquence des variations analogues des éléments de matrice $z_{O E}$ qui déterminent les sections efficaces d'émission photoéleclrique ou de capture des neutrons. Nous verrons que pour les neutrons libres de faible énergie, ces sections sont considérablement accrues si leur énergie correspond à un niveau virtuel. Le phénomène de résonance qui joue dans ce cas est analogue à celui qui se présente pour ia pénétration des particules à travers une barrière de potentiel.

Afin d'obtenir clairement l'ordre de grandeur de ces phénomènes, nous commencerons par étudier le cas schématique d'un potentiel limité constant pour lequel les calculs sont simples. Nous montrerons ensuite comment on peut généraliser les résultats obtenus au cas d'un potentiel quelconque à décroissance rapide.

\section{Section efficace de capture pour un potentiel}

(1) Nous désignons toujours par $S, P, D, \ldots$ les termes appartenant au spectre discontinu et par $s, p, d \ldots$. les termes du spectre continu. 
limité constant. - Soit $-\varepsilon_{0}$ la valeur constante $d u$ potentiel d'interaction $U$ pour une distance $r$ inférieure à la distance $r_{0}$ au delà de laquelle ce potentiel est nul. Par suite de la symétrie sphérique les fonctions d'onde sont de la forme

$$
\Downarrow_{n l m}=\mathscr{P}_{l}^{m}(\cos \theta) \mathbf{e}^{i m_{\varphi}} f_{n l}(r) \mathbf{e}^{\frac{2 \pi i}{h} E_{n l} t} .
$$

$\mathscr{T}_{l}^{m}$ représentant les fonctions de Legendre normalisées.

Les fonctions radiales $f_{n l}(r)$ relatives au potentiel constant limité sont bien connues; nous rappelonsleurs expressions en appendice.

La valeur d'un élément de matrice $z_{n, n^{\prime}}$ relatif à la coordonnée $z$ s'obtient très facilement dans le cas que nous considérons à partir de la relation quantique

$$
\mu \frac{\mathrm{d}^{2} z_{n n^{\prime}}}{\mathrm{d} t^{2}}=-\left(\frac{\partial U}{\partial z}\right)_{n n^{\prime}}=-\left(\frac{\mathrm{d} U}{\mathrm{~d} r} \cos \theta\right)_{n n^{\prime}}
$$

qui donne de suite

$$
\begin{aligned}
& \frac{4 \pi^{2} \mu}{h^{2}}\left(E_{n}-E_{n^{\prime}}\right)^{2}\left|z_{n n^{\prime}}\right| \\
& =(\cos \theta)_{l m l^{\prime} m^{\prime}} \int_{0}^{\infty} r^{2} \frac{\mathrm{d} U}{\mathrm{~d} r} f_{n l}(r) f_{n^{\prime} l^{\prime}}(r) \mathrm{d} r
\end{aligned}
$$

L'élément de matrica relatif à $\cos \theta$ est nul sauf si $m=m^{\prime}, l=l^{\prime} \pm 1$; il a alors pour valeur (en appelant $l$ le plus petit des deux nombres quantiques azimu$\operatorname{taux})$

$$
\sqrt{\frac{(l+1)^{2}-m^{2}}{(2 l+1)(2 l+3)}}
$$

Quant à l'intégrale, $U$ étant partout constant sauf au voisinage de $r=r_{0}$ où l'on a

$$
\left\lceil\left.\frac{\mathrm{d} U}{\mathrm{~d} r}\right|_{r_{0}^{-}} ^{r_{0}^{+}}=\varepsilon_{0}\right.
$$

elle a pour valeur

$$
\varepsilon_{0} r_{0}^{2} f_{n l}\left(r_{0}\right) f_{n^{\prime} l^{\prime}}\left(r_{0}\right) .
$$

En désignant par v la fréquence qui correspond à la transition envisagée, on a donc

$\left|z_{n n^{\prime}}\right|=\frac{1}{4 \pi^{2} \mu} \frac{\varepsilon_{0}}{y^{2}} \sqrt{\frac{(l+1)^{2}-m^{2}}{(2 l+1)(2 l+3)}} r_{0}{ }^{2} f_{n l}\left(r_{0}\right) f_{n^{\prime} l^{\prime}}\left(r_{0}\right)$.

Capture des neutrons sur un niveau $P$. - Nous appliquerons d'abord cette formule pour calculer, à partir des relations (3) et (6), la section efficace de capture sur un niveau discret $\boldsymbol{P}$ d'un neutron provenant d'une onde libre $s$. En remplaçant les fonctions $f$ correspondantes par leurs expressions [appendice, formules (15), (18) et (19), (21), (22)] et en posant

$$
\begin{aligned}
& f=k^{\prime} r_{0}=2 \pi r_{0} \sqrt{\mu_{\mu}\left(\varepsilon_{0}+\omega\right)}, h \\
& \rho_{0}=k_{0} r_{0}=2 \pi r_{0} \sqrt{2 \mu \varepsilon_{0}} / h
\end{aligned}
$$$$
\beta^{2}=\frac{\varepsilon}{\xi_{0}}
$$

on trouve (1)

$$
\begin{gathered}
\left(\sigma_{c}\right)_{P_{s}}=\frac{4}{3} \frac{2 j_{1}}{2 j_{0}}+1 \frac{h}{c^{3}}\left(\frac{Z e}{\boldsymbol{H}_{0}}\right)^{2} \frac{\left(1+\beta \rho_{0}\right)^{2}}{\beta^{2} \rho_{0}^{2}+3\left(1+\beta_{0}\right)} \\
\times \sqrt{\frac{\hat{\varepsilon}_{0}}{\omega} \frac{\varepsilon_{0}-\varepsilon}{\varepsilon+\omega} \frac{\varepsilon_{0} \sin ^{2} p}{\omega+\varepsilon_{0} \cos ^{2} p} .}
\end{gathered}
$$

Rappelons que $\varepsilon_{0}$ est la profondeur du trou de potentiel, $\varepsilon$ l'énergie de liaison du neutron sur le niveau $\boldsymbol{P}$ considéré (déterminée en fonction de $\varepsilon_{0}$ et de $r_{0}$ par le graphique donné dans l'appendice), et $\omega$ l'énergie cinétique disponible du neutron incident.

La formule (12) indique que la section efficace de capture doit présenter, quand croît l'énergie des neutrons incidents (et par suite p), alternativement des minimums nuls et des maximums de plus en plus petits. Mais elle donne, sauf si $\omega$ est très petit par rapport à $\varepsilon_{0}$, des valeurs absolues très faibles, de l'ordre de $10^{-30} \mathrm{~cm}^{2}$, qui rendraient pratiquement inobservable le phénomène correspondant. La première difficulté signalée au début de cet article. relativement à la probabilité de capture simple des neutrons rapides, subsiste donc.

Quand l'énergie $\omega$ tend vers zéro la section efficace donnée par la formule (12) augmente indéfiniment comme l'inverse de la vitesse des neutrons incidents. Mais mème pour des neutrons n'ayant que l'énergie d'agitation thermique ordinaire, la valeur de $\sigma_{c}$ n'est encore en général que de l'ordre de $10^{-20} \mathrm{~cm}^{2}$.

Cependant s'il se trouve que pour des neutrons lents $\cos p$ soit nul, le dénominateur du dernier facteur de l'expression (12) se réduira à $\omega$, au lieu d'être de l'ordre de grandeur de $\varepsilon_{0}$. La section efficace de capture sera donc très augmentée. Le dénominateur qui intervient ici provient de la normalisation de l'onde $s$ du spectre continu dans l'intérieur du trou de potentiel, et la condition $\cos \rho=0$ est celle qui détermine les niveaux $s$ virtuels. La grande augmentation de $\sigma_{c}$ est donc due a un phénomène de résonance se produisant quand un de ces niveaux virtuels coïncide avec l'éner gie des neutrons incidents.

Notamment si l'on a

$$
\cos r_{0}\left(=\cos k_{0} r_{0}=\cos \frac{2 \pi r_{0}}{h} \sqrt{2 \mu \varepsilon_{0}}\right)=0
$$

c'est-à-dire s'il existe un état $S$ correspondant à une énergie de liaison nulle du neutron, la résonance se produira pour les neutrons infiniment lents. La section $\tau_{c}$ augmentera alors comme l'inverse du cube de la vitesse des neutrons incidents $\left({ }^{2}\right)$. Numériquement, on trouve dans ce cas (en tenant compte du fait que le

(') Cf F. Perrin, W. M. Elsasser. Loc. cit., p. 194.

(2) La section efficace $\left(\sigma_{c}\right) P_{s}$ ne peut pas être supérieure à $\frac{\Lambda^{2}}{4 \pi}$, $\triangle$ étant la longueur d'onde des neutrons incidents; l'expression de $\left(\sigma_{c}\right) P_{s}$ donnée ici croissant comme $\Lambda^{3}$ finirait par dépasser cette limite, mais alors la condition (i) ne serait plus satisfaite et et les formules utilisées ne seraient pas valables. 
nombre atomique $Z$ est de l'ordre de la moitié du nombre de masse) que l'on a environ

$$
\left(\sigma_{c}\right)_{P_{s}} \sim\left(\frac{\varepsilon_{0}}{\omega}\right)^{\frac{3}{2}} 10^{-30} \mathrm{~cm}^{2}
$$

avec (pour le premier cas possible de résonance)

$$
\varepsilon_{0} \sim \frac{\check{s}}{r_{0}{ }^{2}} 10^{-24} \text { MVe. }
$$

En prenant $r_{0}=5.10^{-13} \mathrm{~cm}$ on aura $\Xi_{0}=20 \mathrm{MVe}$, et l'on obtient, pour la résonance exacte et des neutrons ayant l'énergie d'agitation thermique, une section efficace de capture de l'ordre de $10^{-18} \mathrm{~cm}^{2}$.

Si la résonance n'est qu'approchée, on aura, en posant $\cos f_{0}=r_{1}$,

$$
\left(\delta_{c}\right)_{P_{s}} \sim \frac{\varepsilon_{0}}{\omega+r_{i}{ }^{2} \hat{\varepsilon}_{0}}\left(\frac{\varepsilon_{0}}{\omega}\right)^{\frac{1}{2}} \cdot 10-30 \mathrm{~cm}^{2}
$$

et pour les valeurs de $\omega$ petites par rapport à $r_{1}^{2} \hat{s}_{0}$

$$
\left(\sigma_{c}\right)_{P_{s}} \sim \frac{1}{\eta_{i}^{2}}\left(\frac{\Xi_{0}}{\omega}\right)^{\frac{1}{2}} \cdot 10^{-30} \mathrm{~cm}^{2} .
$$

La section efficace sera donc proportionnelle, pour les neutrons lents, à l'inverse de leur vitesse.

Une résonance approchée avec クde l'ordre de un centième suffit pour donner une section efficace de capture de l'ordre des plus grandes observées. si l'on admet que les neutrons ralentis par les substances hydrogénées ont une énergie de l'ordre de l'énergie thermique, et cela en supposant toujours une interaction limitée à une distance inférieure à $5 \cdot 10^{-13} \mathrm{~cm}$.

Capture des neutrons sur un niveau $S$. - Pour qu'un neutron puisse être capturé sur un niveau $S$ il faut qu'il provienne d'une onde libre $p$. Les formules (3), (6), (10) et les formules (11), (14), (23), (25), (26) de l'appendice donnent dans ce cas

$$
\begin{aligned}
\left(\sigma_{c}\right)_{S_{p}}= & \frac{4}{3} \frac{2 j_{1}+1}{2 j_{0}+1} \frac{h}{c^{3}}\left(\frac{Z e}{M_{0}}\right)^{2} \frac{\beta}{1+\beta \rho_{0}} \times \sqrt{\frac{\omega}{\varepsilon_{0}} \frac{\varepsilon_{0}-\varepsilon}{\varepsilon+\omega}} \\
& \frac{\varepsilon_{0}^{2}(\sin \rho-\rho \cos \rho)^{2}}{\left(\varepsilon_{0} \sin \rho+\omega \rho \cos \rho\right)^{2}+\omega\left(\varepsilon_{0}+(1)\right) \rho^{2} \sin ^{2} q} .
\end{aligned}
$$

Cette formule donne en général des valeurs très petites pour $\sigma_{c}$, et tendant vers 0 quand $\omega$ tend vers zéro. Cependant quand $\omega$ est petit (neutrons lents) il se produit un phénomène de résonance rendant $\sigma_{c}$ grand si un état virtuel $p$ se trouve avoir une énergie égale à $\omega$, ce qui correspond à la condition

$$
\varepsilon_{0} \sin \rho+\omega \rho \cos \rho=0 .
$$

On a alors approximativement

$$
\left(\sigma_{c}\right)_{S_{p}} \sim\left(\frac{\varepsilon_{0}}{\omega}\right)^{\frac{5}{2}} \cdot 10^{-31} \mathrm{~cm}^{2}
$$

Mais cette résonance est très aiguë; elle ne s’étend que sur un intervalle d'énergie de l'ordre de

$$
\delta \omega=\omega \sqrt{\frac{\omega}{\varepsilon_{0}}}
$$

et il est très peu probable qu'un niveau virtuel existe pour une valeur suffisamment petite de $\omega$ pour que $\left(\sigma_{c}\right)_{S p}$ devienne observable.

Capture d'un neutron avec projection d'une particule matérielle. - Nous avons vu que le phénomène de résonance qui détermine les grandes sections efficaces pour la caplure simple des neutrons lents provient du facteur d'intensité de l'onde incidente dans l'intérieur du noyau. La résonance doit donc aussi déterminer de grandes sections efficaces si la capture du neutron lent est accompagnée de l'émission d'une parlicule matérielle (proton, ou hélion) au lieu d'un photon. Il n'y a d'ailleurs plus de règle de sélection dans ce cas, et l'existence d'un niveau $P$ libre ne sera pas nécessaire.

Mais il faudra que la réaction dégage suffisamment d'énergie pour que la barrière de potentiel n'empêche pas l'émission de la particule chargée; c'est donc sans doute pour les éléments de petit nombre atomique qu'il peut y avoir dans ce cas de grandes sections efficaces. Or Chalwick a justement montré que les deux éléments légers fortement absorbants pour les neutrons lents, le bore et lithium, émettent, lors de la capture, des particules matérielles.

Pour une capture avec émission matérielle on peut admettre, que par suite du fort couplage entre les particules nucléaires et le neutron, la section efficace de capture aura comme ordre de grandeur le produit de la section efficace maximum correspondant à l'onde $s$ de la décomposition en ondes sphériques de l'onde plane incidente, e'est-à-dire ( $\left.{ }^{1}\right)$

$$
\frac{1}{4 \pi} \Lambda^{2}=\frac{h^{2}}{8 \pi \mu} \frac{1}{\omega}
$$

multipliée par l'Intensité relative de l'onde $s$ dans l'intérieur du trou de potentiel, c'est-à-dire par

$$
\frac{b^{\prime 2}}{b^{2}}=\frac{\omega}{\omega+\varepsilon_{0} \cos ^{2} p} .
$$

On obtient ainsi

$$
\tau_{c} \sim \frac{h^{2}}{8 \pi \mu} \frac{1}{\omega+\varepsilon_{0} \cos ^{2} \rho}=\pi r_{0}^{2} \frac{1}{\rho_{0}^{2}} \frac{\varepsilon_{0}}{\omega+\varepsilon_{0} \cos ^{2} p}
$$

Si la particule émise avait à franchir une barrière de potentiel il faudrait mulplier cette expression par le coefficient de transparence correspondant. Cette formule grossière donne une section efficace de l'ordre de la section nucléaire $\pi r_{0}{ }^{2}$, sauf si la condition de résonance $\cos p=0$ est satisfaite. Pour une résonance approchée $\left(\cos p=\eta_{i}\right)$ on aura pour les neutrons lents une section

(1) N. F. Mort. Proc. Roy. Soc., A, 1931, 133, p. 228. 
No 5. THẾORIE DE LA CAPTURE SÉLECTIVE DES

efficace grande et sensiblement indépendante de leur vitesse

$$
\sigma_{c} \sim \pi r_{0}^{2}-\frac{1}{r_{i}^{2} p^{2}} .
$$

Cette section sera de l'ordre de 10-91 $\mathrm{cm}^{2}$ si $\eta$ est d'environ un centième. Il se peut que par hasard une telle condition soit satisfaite pour les deux éléments légers bore et lithium (voir note p. 202).

Existence du phénomène de résonance pour un potentiel limité quelconque. - Nous supposerons seulement une décroissance assez rapide du potentiel d'interaction, fonction toujours de la distance seule, pour qu'on puisse le considérer comme nul en dehors d'une sphère $\Sigma$ de rayon $r_{0}$.

A l'intérieur de la sphère $\Sigma$, l'équation d'onde radiale n'admet qu'une solution $f(r)$ régulière à l'origine, déterminée à un facteur près. A l'extérieur de la sphère ', le potentiel étant nul, on a

$$
f=\frac{a}{\sqrt{ } r} J_{l+\frac{1}{2}}(k r)+\frac{b}{\sqrt{r}} J_{-l-\frac{1}{2}}(k r)
$$

et si l'on normalise la fonction pour un spectre en énergie, on aura

$$
a^{2}+b^{2}=\frac{4 \pi^{2} \mu}{h^{2}}
$$

A l'intérieur de la sphère $\mathbf{\Sigma}$ nous poserons

$$
f=\Lambda \psi(r)
$$

de façon à pouvoir normaliser dans ce domaine la fonction d'onde comme dans le cas d'un spectre discontinu par la condition

$$
\int_{0}^{r_{0}} \psi^{2} r^{2} \mathrm{~d} r=1
$$

L'intérêt d'introduire le facteur $\Lambda$ est le suivant : Dans les formules de probabilité de transition entre un état du spectre discontinu et un état du spectre continu apparait en facteur le carré de l'élément de matrice $\mathrm{du}$ rayon vecteur $\left(r_{O E}\right)^{2}$ calculé pour des fonctions d'onde du spectre continu normalisées par rapport à un élément différentiel de représentation. Les fonctions d'onde des états discrets étant pratiquement nulles à l'extérieur de $\Sigma$, ce facteur sera égal à $\Lambda^{2}\left|R_{O E}\right|^{2}, R_{O E}$ étant un élément de matrice quasi discret, calculé à partir de la fonction $\psi$, ayant le même ordre de grandeur que les éléments de matrice relatifs à des transitions entre deux états du spectre discontinu, et pouvant par suite ètre estimé indépendamment de l'étude du spectre continu. Les phénomènes de résonance apparaitront entièrement dans les variations du facteur $\Lambda^{2}$ que nous allons évaluer.

Les conditions de continuité à la surface de la sphère $\Sigma$ donnent

$$
\begin{aligned}
& \frac{a}{\sqrt{r_{0}}} J_{l+\frac{1}{2}}\left(k r_{0}\right)+\frac{b}{\sqrt{r_{0}}} J_{-l-\frac{1}{2}}\left(k r_{0}\right)=\Lambda \psi\left(r_{0}\right) \\
& \left.\begin{array}{rl}
a \frac{\mathrm{d}}{\mathrm{d} r}\left[\frac{\mathrm{1}}{\sqrt{r}} J_{l+\frac{1}{2}}(k r)\right]_{r}=r_{0} & +b \frac{\mathrm{d}}{\mathrm{d} r}\left[\frac{\mathrm{1}}{\sqrt{r}} J_{\left.-l-\frac{1}{2}(k r)\right]_{r=r_{0}}}\right\} \\
=\Lambda \frac{\mathrm{d}}{\mathrm{d} r} \psi(r)_{r=r_{0}} .
\end{array}\right\}
\end{aligned}
$$

Pour abréger nous omettrons d'écrire dans la suite les arguments $k r_{0}$ ou $r_{0}$ des fonctions. Une transformation simple des équations précédentes donne

$$
\begin{aligned}
& a J_{l+\frac{1}{2}}+b J_{-l-\frac{1}{2}}=\Lambda V r_{0} \psi \\
& a J_{l+\frac{1}{2}}-b J_{-l+\frac{1}{2}}=\Lambda \frac{\sqrt{r_{0}}}{k}\left(\psi^{\prime}+\frac{l+1}{r_{0}} \psi\right) .
\end{aligned}
$$

Le déterminant de ce système linéaire en $a$ et $b$ est

$$
\begin{aligned}
-J_{l+\frac{1}{2}} J-l+\frac{1}{2}-J_{l-\frac{1}{2}} J-l-\frac{1}{\underline{2}} & =-\frac{2 \sin \left(l+\frac{1}{2}\right) r}{\pi k r_{0}} \\
& =\frac{2(-1)^{l+1}}{\pi k r_{0}}
\end{aligned}
$$

et l'on obtient

$$
\left.\begin{array}{l}
\boldsymbol{a}=\frac{\Lambda(-1)^{l} \pi \sqrt{r_{0}^{3}}}{2}\left[k \psi_{-l+\frac{1}{2}}+\left(\psi^{\prime}+\frac{l+1}{r_{0}} \psi\right) J_{-l-\frac{1}{2}}\right] \\
b=\frac{\Lambda(-1)^{l} \pi \sqrt{r_{0}^{3}}}{2}\left[k \psi J_{l-\frac{1}{2}}-\left(\psi^{\prime}+\frac{l+1}{r_{0}} \psi\right) J_{l+\frac{1}{2}}\right]
\end{array}\right\}
$$

En portant ces valeurs dans la condition de normalisation (22) on trouve

$$
\begin{aligned}
\Lambda^{2}= & \frac{16 \mu}{h^{2} r_{0}^{3}} \frac{1}{J_{-l-\frac{1}{2}}^{2}}\left[\left(\psi^{\prime}+\frac{l+1}{r_{0}} \psi+\frac{-l+\frac{1}{2}}{J-l-\frac{1}{2}} k \psi\right)^{2}\right. \\
+ & \frac{J^{2}+\frac{1}{2}}{J_{-l-\frac{1}{2}}^{2}}\left(-\psi^{\prime}-\frac{l+1}{r_{0}} \cdot+\frac{l-\frac{1}{2}}{J} k \psi\right)^{-1}
\end{aligned}
$$

Lorsque $l$ est nul, ce qui est le cas le plus important, cette iormule se réduit à

$$
\Lambda^{2}=\frac{8 \pi \mu}{h^{2}} \frac{k}{k^{2} r_{0}^{2} \psi^{2}+\left(r_{0} \psi^{\prime}+\psi\right)^{2}} .
$$

Quand $k r_{1}$ est petit, la lonction $\psi$ sera sensiblement indépendante de $k$ si le potentiel $U$ devient rapidement grand, en valeur absolue, quand $r$ décroit en dessous de $r_{9}$. L'expression de $\Lambda^{2}$ montre alors l'existence de maxima de résonance très prononcés, déterminés par 
la condition $r_{0} \psi^{\prime}=-\psi$. Cette condition aux limites est satisfaite pour une série de valeurs propres de l'énergie; il y aura résonance si l'une de ces valeurs est presque nulle. On peut remarquer l'analogie de la formule avec une formule de dispersion.

Lorsque $l$ est différent de zéro il se produira aussi un phénomène de résonance pour $k$ petit si la première parenthèse entre crochets dans la formule (23̈) s'annule :

$$
\psi^{\prime}+\frac{l+1}{r_{0}} \psi+\frac{J_{-l-\frac{1}{2}}}{J} k \psi=0 .
$$

On a alors $a=0$ et la première équation (23) donne

$$
\Lambda_{\max }^{2}=\frac{4 \pi^{2} \mu}{h^{2} r_{0} \psi^{2}} J_{-l-\frac{1}{2}}^{2}\left(k r_{0}\right)
$$

$k$ étant petit cette expression est proportionnelle à $k^{-2 l-1}$; elle est donc d'un ordre de grandeur d'autant plus grand que $l$ est plus grand, mais la bande de résonance devenant beaucoup plus étroite, l'effel total de résonance devient de plus en plus négligeable.

\section{Appendice.}

Particule mobile dans un potentiel sphérique constant limité. - Pour un potentiel d'interaction défini par

$$
\left.\begin{array}{ll}
U(r)=0 & r>r_{0} \\
U(r)=-\varepsilon_{0} & r<r_{0} \quad\left(\varepsilon_{0}>0\right)
\end{array}\right\}
$$

l'équation différentielle relative à la partie radiale de la fonclion d'onde est

$$
\frac{\mathrm{d}^{2} f}{\mathrm{~d} r^{2}}+\frac{2}{r} \frac{\mathrm{d} f}{\mathrm{~d} r}+\left(x-\frac{l(l+1)}{r^{2}}\right) f=0
$$

$l$ étant le nombre quantique azimutal, et la constante $\alpha$ ayant suivant les cas les valeurs suivantes :

Pour la particule liée (énergie négative $E=-\varepsilon$ ).

$$
\left.\begin{array}{ll}
\alpha=-\frac{8 \pi^{2} \mu}{h^{2}} \varepsilon=-K^{2} & r>r_{0} \\
x=\frac{8 \pi^{-} \mu}{h^{2}}\left(\varepsilon_{0}-\Xi\right)=K^{\prime 2} & r<r_{0} .
\end{array}\right\}
$$

Pour la particule libre (énergie positive $E=(\prime)$ )

$$
\left.\begin{array}{ll}
\alpha=\frac{8 \pi^{2} \mu}{h^{2}} \omega=k^{2} & r>r_{0} \\
\alpha=\frac{8 \pi^{2} \mu}{h^{2}}\left(\varepsilon_{0}+\omega\right)=k^{\prime 2} & r<r_{0} .
\end{array}\right\}
$$

Nous poserons de plus

$$
\frac{8 \pi^{2} \mu_{3}}{h^{2}} \varepsilon_{0}=K^{2}+K^{\prime 2}=k^{\prime 2}-k^{2}=k_{0}^{2}
$$

La fonction $f$, qui doit ètre bornée et continue ainsi que sa dérivée pour $r=r_{0}$, s'exprime simplement dans les différents cas :

Pour la particule liée, on a

$$
\left.\begin{array}{ll}
f(r)=A \frac{1}{\sqrt{\mathrm{i} r}} H_{l+\frac{1}{9}}^{1}(\mathrm{i} K r) & r>r_{0} \\
f(r)=A^{\prime} \frac{1}{\sqrt{r}} J_{l+\frac{1}{2}}\left(K^{\prime} r\right) & r<r_{0}
\end{array}\right\}
$$

$H^{\prime} l+\frac{1}{2}$ étant la fonction de Hankel de première espèce d'ordre $l+\frac{1}{2}$, et $J_{l+\frac{1}{2}}$ la fonction de Bessel de mème ordre. Les conditions de continuité ne sont compatibles dans ce cas que si $k_{0} r_{0}$ est suffisamment grand, et seulement pour certaines valeurs en nombre fini de l'énergie de liaison $\varepsilon$ (spectre discontinu fini). Elles déterminent alors le rapport des constantes $\mathrm{A}$ et $\mathrm{A}^{\prime}$ qu'on achève de déterminer par la condition de normalisation

$$
\int_{0}^{\infty} f(r)^{2} r^{2} \mathrm{~d} r=1 .
$$

Pour la particule libre, on a

$$
\left.\begin{array}{ll}
f(r)=\frac{a_{1}}{\sqrt{r}} J_{l+\frac{1}{2}}(k r)+\frac{a_{2}}{\sqrt{r} J_{-l-\frac{1}{2}}(k r)} & r>r_{0} \\
f(r)=\frac{a^{\prime}}{\sqrt{r}} J_{l+\frac{1}{2}}\left(k^{\prime} r\right) & r<r_{0} .
\end{array}\right\}
$$

Les conditions de continuité peuvent (lans ce cas ètre satisfailes quelle que soit l'énergie $\omega$ (spectre continu); elles déterminent les rapport $a_{1}: a^{\prime}$ et $a_{2}: a^{\prime}$. La normalisation des fonctions $f$ relatives à ce spectre continu représenté en fonction de $k$ s'obtient par la condition

$$
\int_{0}^{\infty} f(r, k) r^{2} \mathrm{~d} r \int_{k-\Delta k}^{k+\Delta k} f(r, u) \mathrm{d} u=1
$$

qui donne

$$
\left(a_{1}^{2}+{a_{2}^{2}}^{2}\right)_{k}=k .
$$

On en en déduit la normalisation pour la représentalion du spectre continu en fonction de l'énergie en divisant la fonction $f$ obtenue par la racine carrée de la dérivée de $\omega$ par rapport à $k$ ce qui donne

$$
\left(a_{1}^{2}+a_{2}^{2}\right)_{\ldots}=\frac{4 \pi^{2} j}{h^{2}} .
$$

Etats stationnaires discrets $\mathbf{s}$. - Pour la particule liée dans un état $s(l=0)$, on a 


$$
\left.\begin{array}{ll}
f_{S}=B \frac{\mathbf{e}^{-K r}}{r} & r>r_{0} \\
f_{S}=B^{\prime} \frac{\sin K^{\prime} r}{r} & r<r_{0} .
\end{array}\right\}
$$

Les conditions de continuité s'écrivent

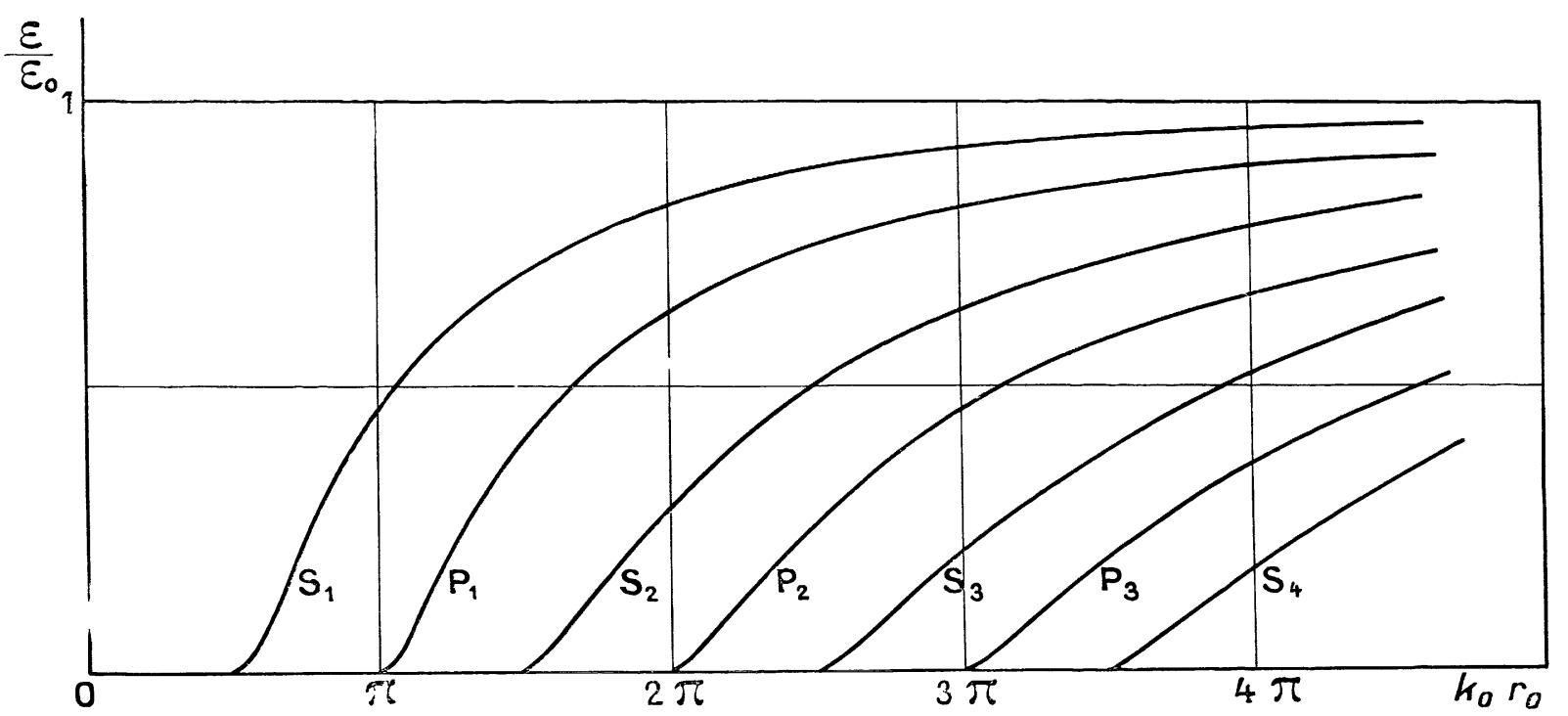

Fig. 1.

Ces relations permettent une détermination graphique simple de l'énergie $\varepsilon_{S}$. Il n'y a pas d'état stationnaire possible si $k_{0} r_{0}$ est inférieur à $\pi / 2$, il y en a un si $k_{0} r_{0}$ est compris entre $\tau / 2$ et $3 \pi / 2$, et $n$ si $k_{0} r_{0}$ est compris entre $(2 n-1) \pi / 2$ et $(2 n+1) \pi / 2$. La figure 1 donne les valeurs de $\varepsilon_{S}: \varepsilon_{0}$ en fonction de $k_{0} r_{0}$ pour les premiers étals $S$.

La normalisation des fonctions $f_{S}$ donne en tenant compte des relations $(\mathbf{1 2})$,

$B^{2}=2 \frac{K^{\prime 2}}{\Lambda^{2}+K^{\prime 2}} \frac{K \mathbf{e}^{2} K^{\prime \prime}}{1+K r_{0}}, \quad B^{\prime 2}=2 \frac{K}{1+K r_{0}}$.

Etats stationnaires discrets P. - Pour la particule liée dans un état $P(l=1)$, on a

$$
\left.\begin{array}{ll}
f_{P}=C \frac{1+K r}{r^{2}} \mathrm{e}^{-K r} & r>r^{0} \\
f_{P}=C^{\prime} \frac{\sin K^{\prime} r-K^{\prime} r \cos K^{\prime} r}{r^{2}} & r<r_{0}
\end{array}\right\}
$$

Les conditions de continuité s'écrivent

$$
\left.\begin{array}{rl}
C\left(\mathbf{I}+K r_{0}\right) \mathbf{e}^{-K r_{0}} & =C^{\prime}\left(\sin K^{\prime} r_{0}-K^{\prime} r_{0} \cos K^{\prime} r_{0}\right) \\
\quad-C K^{2} \mathbf{e}^{-K r_{0}} & =C^{\prime} K^{\prime 2} \sin K^{\prime} r_{01}
\end{array}\right\}
$$

d'où, en posant $X^{\prime}=K^{\prime} r_{0}$,

$$
Y=X \cot X \quad Y=1+\frac{K^{\prime 2}}{K^{2}}+\frac{K^{\prime}}{K} X
$$

relations qui permettent encore une détermination graphique simple des énergies $\varepsilon_{p}$. Il n'y a pas d'état stationnaire $\boldsymbol{P}$ si $k_{0} r_{0}$ est inférieur à $\pi$, il y en a un si $k_{0} r_{0}$ est entre $\pi$ et $2 \pi$, et $n$ si $k_{0} r_{0}$ est entre $n \pi$ et $(n+1) \pi$. Les valeurs de $\varepsilon_{P}: \varepsilon_{o}$ sont domnées en fonction de $k_{0} r_{0}$ sur la figure 1 , pour les premiers niveaux $P$.

La normalisation des fonctions $f_{p}$ donne en tenant compte des relations $(\mathbf{1} \overline{7})$,

$$
\left.\begin{array}{l}
C^{2}=2 \mathrm{e}^{2 K r_{0}} r_{0} \frac{K^{\prime 2}}{K^{2}+K^{\prime 2}} \frac{1}{K^{2} r_{0}{ }^{2}+3\left(1+K r_{0}\right)} \\
C^{\prime 2}=2 \frac{1}{K^{\prime / 4} r_{0}} \frac{K^{2}+K^{\prime 2}\left(1+K r_{0}\right)^{2}}{K^{2} r_{0}{ }^{2}+3\left(1+K r_{0}\right)} .
\end{array}\right\}
$$

Spectre continus. - L'onde stationnaire die moment nul $(l=0)$ relative à une valeur positive $\omega$ de l'énergie peut ètre représentée en introduisant un déphasage $\hat{o}_{s}$ :

$$
\left.\begin{array}{ll}
f_{s}=b \frac{\sin \left(k r+\delta_{s}\right)}{r} & r>r_{0} \\
f_{s}=b^{\prime} \frac{\sin k^{\prime} r}{r} & r<r_{0} .
\end{array}\right\}
$$

Les conditions de continuité

$$
\left.\begin{array}{c}
b \sin \left(k r_{0}+\grave{o}_{s}\right)=b^{\prime} \sin k^{\prime} r_{0} \\
b k \cos \left(k r_{0}+\delta_{s}\right)=b^{\prime} k^{\prime} \cos k^{\prime} r_{0}
\end{array}\right\}
$$




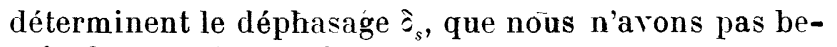
soin de connaitre, et le rapport

$$
\frac{b^{\prime 2}}{b^{2}}=\frac{k^{2}}{k^{2} \sin ^{2} k^{\prime} r_{0}+k^{\prime 2} \cos ^{2} k^{\prime} r_{0}} .
$$

La normalisation pour un spectre en énergie se déduit de la relation générale $(10)$, qui donne

$$
b_{\omega}^{2}=\frac{2}{\pi k}\left(a_{1}^{2}+a_{2}^{2}\right)_{\omega}=\frac{8 \pi \mu}{h^{2}} \frac{1}{k}
$$

et l'on obtient ${\boldsymbol{b}^{\prime}}_{\omega}^{2}$ à l'aide de la relation $(\boldsymbol{2 1})$.

Spectre continu p. - Pour $l=1$, on a, en introduisant encore un déphasage pour l'onde extérieure,

$$
\begin{array}{ll}
f_{p}=c \frac{\sin \left(k r+\delta_{p}\right)-k r \cos \left(k r+\delta_{p}\right)}{r^{2}} & r>r_{0} \\
f_{p}=c^{\prime} \frac{\sin k^{\prime} r-k^{\prime} r \cos k^{\prime} r}{r^{2}} & r<r_{c}
\end{array}
$$

Les conditions de continuité

$$
\begin{aligned}
c\left[\sin \left(k r_{0}+\delta_{p}\right)\right. & \left.-k r_{0} \cos \left(k r_{0}+\delta_{p}\right)\right] \\
& =c^{\prime}\left(\sin k^{\prime} r_{0}-k^{\prime} r_{0} \cos k^{\prime} r_{0}\right) \\
c k^{2} \sin \left(k r_{0}+\delta_{p}\right) & =c^{\prime} l^{\prime 2} \sin k^{\prime} r_{0}
\end{aligned}
$$

déterminent le déphasage $\delta_{p}$, et le rapport

$\frac{c^{\prime 2}}{c^{2}}=\frac{k^{6} r_{0}{ }^{2}}{\left(k_{0}^{2} \sin k^{\prime} r_{0}+k^{2} k^{\prime} r_{0} \cos k^{\prime} r_{0}\right)^{2}+k^{2} k^{\prime \prime 4} r_{0}{ }^{2} \sin ^{2} k^{\prime} r_{0}}$.

La normalisation pour un spectre en énergie donne ici

$$
c^{2}=\frac{8 \pi \mu}{h^{2}} \frac{1}{h^{3}}
$$

et la relation (27) donne alors $c^{\prime 2}$.

Note ajoutée sur épreuves. - Lévalualion faite page 198 pour la capture d'un neutron avec émission d'une autre particule est incorrecte. Dans ce cas l'onde neutronique pénétrant dans le noyau disparaît par absorption. La section efficace maximum $\Lambda^{2}: 4 \pi$ doil lonc être réluite seulement par la réflexion partielle sur la surface du noyau. On trouve ainsi, pour les neutrons lents, une section efficace de l'ordre de $\frac{h^{2}}{2 \pi \mu} \frac{1}{\sqrt{\varepsilon_{0} \omega}}$, ce qui explique les grandes sections observées pour le lithium et le bore sans phénomène de résonance (cf. F. Perrin, $C . R$. $A c$. Sc., 200, séance du 20 mai 1934). 